\title{
Experimental evidence of turbulent transport regulation by zonal flows
}

\section{G. Birkenmeier}

E-mail: birkenmeier@ipf .uni-stuttgart.de

Institut für Plasmaforschung, Universität Stuttgart, Pfaffenwaldring 31, 70569 Stuttgart, Germany

Max-Planck-Institut für Plasmaphysik, EURATOM-Assoziation, Boltzmannstr.2, 85748 Garching, Germany

\section{Ramisch}

Institut für Plasmaforschung, Universität Stuttgart, Pfaffenwaldring 31, 70569

Stuttgart, Germany

\section{B. Schmid}

Institut für Plasmaforschung, Universität Stuttgart, Pfaffenwaldring 31, 70569

Stuttgart, Germany

\section{U. Stroth}

Institut für Plasmaforschung, Universität Stuttgart, Pfaffenwaldring 31, 70569 Stuttgart, Germany

Max-Planck-Institut für Plasmaphysik, EURATOM-Assoziation, Boltzmannstr.2, 85748 Garching, Germany

\begin{abstract}
The regulation of turbulent transport by zonal flows is studied experimentally on a flux surface of the stellarator experiment TJ-K. Data of 128 Langmuir probes at different toroidal and poloidal positions on a single flux surface enable to measure simultaneously the zonal flow activity and the turbulent transport in great detail. A reduction of turbulent transport by $30 \%$ during the zonal flow phase is found. It is shown that the reduction process is initiated by a modification in the cross phase between density and electric field followed by a reduction in the fluctuation levels, which sustain low transport levels on larger time scales than the zonal flow life time.
\end{abstract}

PACS numbers: 52.25.Xz, 52.25.Fi, 52.35.Ra, 52.55.Hc 
In magnetically confined toroidal plasmas, zonal flows $(\mathrm{ZF})$ are long-living $(\omega \approx 0)$, radially localized $\left(k_{r} \neq 0\right)$, and poloidally and toroidally homogenous $\left(k_{\theta}=k_{\varphi}=0\right)$ potential structures giving rise to a macroscopic $E \times B$-flow [1]. Due to the twodimensional nature of plasma turbulence in the plane perpendicular to the magnetic field of toroidally confined plasmas, the energetics obey an inverse cascade [2]. Consequently, the ZFs are supposed to gain energy from small-scale drift-wave turbulence by a self-organizing process which was described theoretically [3], observed in nonlinear turbulence simulations $[4,5,6]$, and investigated experimentally in detail $[7,8,9,10,11]$. It was shown that the flux surface average of the radial derivative of the Reynolds stress $\left\langle\tilde{v}_{r} \tilde{v}_{\theta}\right\rangle$ is the driving term for ZFs, and that the energy is transferred non-locally in $k$-space, i.e. small-scale eddies release their energy directly to the large scale flows most efficiently by a vortex-thinning process $[9,12]$. In this way, the ZF (predator) is able to reduce turbulence (prey) leading to an interplay between ZFs and turbulence amplitudes as it is described in several types of predator-prey models $[13,14,15]$. These models have attracted attention since they are able to describe many aspects of the experimentally observed $[16,17,18]$ transition from low (L-mode) to high (H-mode) confinement regimes in fusion devices including an oscillatory intermediate phase [19].

The measurements reported in this Letter go beyond these results, and show for the first time the predator-prey-dynamics between ZFs and total turbulent particle transport. It is found (i) that the total turbulent transport is reduced significantly ( $30 \%$ ) during the ZF phase stressing the relevance of ZFs for the global confinement of toroidal plasmas, and (ii) that the reduction is based on modifications of the cross phase between density and electric field fluctuations at the relevant spatial scales rather than on a reduction of corresponding fluctuation levels.

In order to study the interaction of ZFs with turbulence, one has to resolve small spatial and temporal scales of turbulence on the one hand, and, simultaneously, the $\mathrm{ZF}$ on global scales on the other hand. In the stellarator experiment TJ-K [20], this is accomplished by use of two poloidal probe arrays [21]. The arrays are located at different toroidal positions (Top Port Array at $\varphi=230$, Fig. 1a, and Outer Port Array at $\varphi=90$, Fig. 1b) providing Langmuir-probe data from 128 positions on a single flux surface at $\rho=0.5$ (toroidal flux coordinate). The probe tips are equidistantly separated by $d x=0.81 \mathrm{~cm}$ in poloidal direction, and the radial misalignment with respect to the flux surface is smaller than $5 \mathrm{~mm}$ which is below the typical size of turbulent structures of about 3 to $5 \mathrm{~cm}$ [30]. Measurements were carried out with a time resolution of $1 \mathrm{MHz}$ and 1024000 recorded time points per probe in microwave heated [22] helium plasmas with a central density of $n=2.3 \times 10^{17} \mathrm{~m}^{-3}$ and an electron temperature of $T_{e}=8$ $\mathrm{eV}$ (ions are cold [23]) at an on-axis magnetic field of $B=72 \mathrm{mT}$. By simultaneous acquisition of one ion-saturation current signal $\tilde{I}_{\mathrm{i}, \mathrm{sat}}^{j}(t)$ from probe $j$ and two floatingpotential signals $\tilde{\phi}_{\mathrm{fl}}^{j \pm 1}(t)$ from poloidally adjacent probes $j \pm 1$, the local time-dependent turbulent transport $\Gamma_{\text {loc }}^{j}(t)$ at probe $j=1,3,5, .$. is calculated according to

$$
\Gamma_{\mathrm{loc}}^{j}(t)=\tilde{n}(t) \tilde{v}_{r}(t)=\tilde{n}(t) \cdot \frac{\tilde{E}_{\theta}(t)}{B}
$$



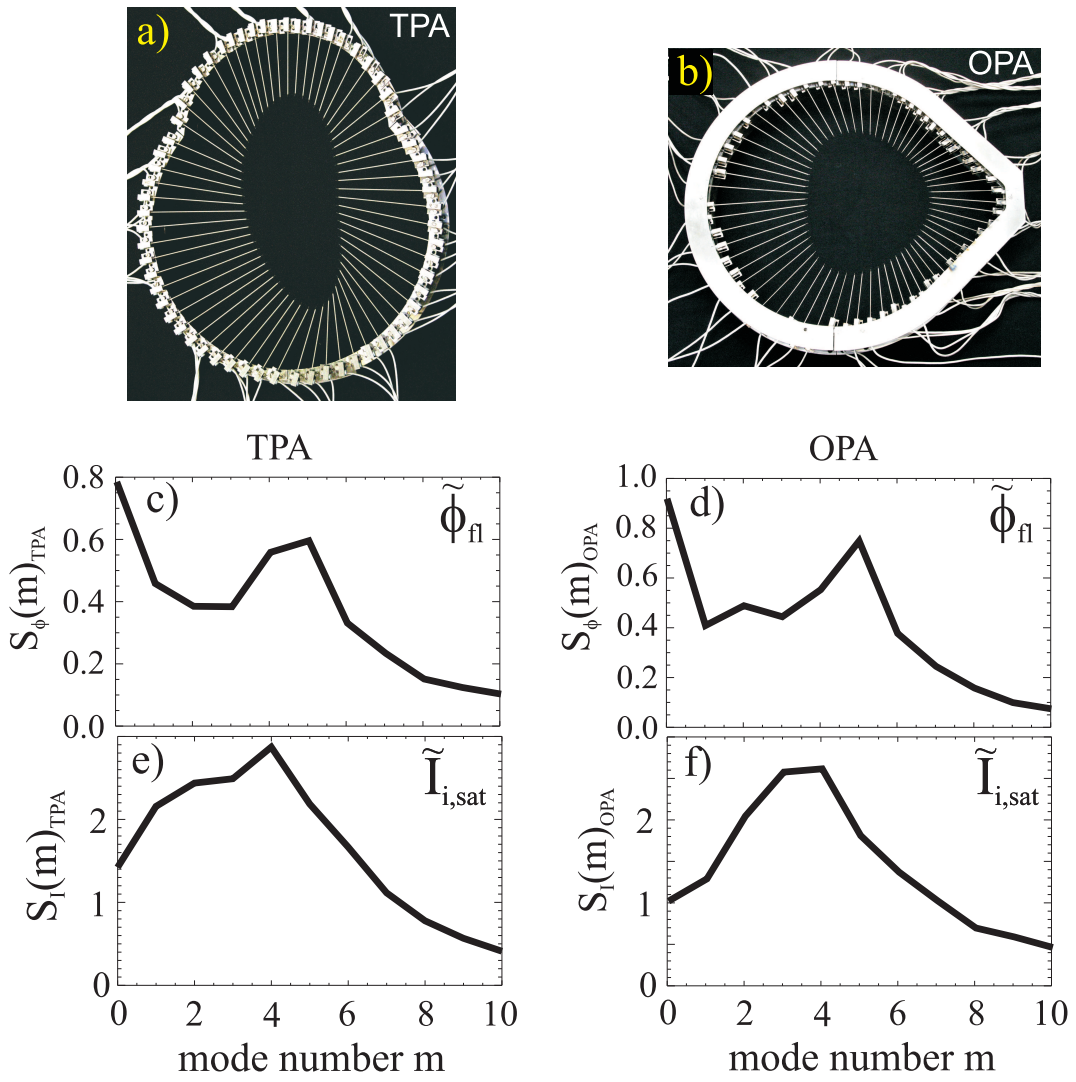

Figure 1. Poloidal power spectra of the floating-potential (c and d) and ion-saturation current fluctuations (e and f) acquired with the Top Port Array (TPA, left) and the Outer Port Array (OPA, right).

$$
\propto \tilde{I}_{\mathrm{i}, \mathrm{sat}}^{j}(t) \cdot\left(\frac{\tilde{\phi}_{\mathrm{fl}}^{j+1}(t)-\tilde{\phi}_{\mathrm{fl}}^{j-1}(t)}{B 2 d x}\right)
$$

with the local density, fluid velocity and electric field fluctuations $\tilde{n}(t), \tilde{v}_{r}(t)$ and $\tilde{E}_{\theta}(t)$, respectively. Due to a flat temperature profile and a low plasma beta in TJ-K, temperature fluctuations are negligible justifying the proportionalities $\tilde{I}_{\mathrm{i} \text {,sat }} \propto \tilde{n}$ and $\tilde{\phi}_{\mathrm{fl}} \propto \tilde{\phi}_{p}$ where $\tilde{\phi}_{p}$ is the plasma-potential fluctuation [24].

In addition to the acquisition of the turbulent transport, the probe arrangement simultaneously enables the determination of poloidal mode-number spectra from 32 probes of the same type $\left(\tilde{\phi}_{\mathrm{fl}}\right.$ or $\left.\tilde{I}_{\mathrm{i}, \mathrm{sat}}\right)$ at each array for every time step. Figures $1 \mathrm{c}$ to f show time averaged mode-number spectra from probe signals whose temporal mean value was subtracted for every single probe separately in order to eliminate effects from the background profile. At both arrays, the spectra of the floating potential signals $S_{\phi}(m)_{\text {TPA }}$ (Fig. 1c) and $S_{\phi}(m)_{\text {OPA }}$ (Fig. 1d) look similar: They have pronounced local maxima at the mode numbers $m=4$ to 5 corresponding to the typical dominant turbulent spatial scales in TJ-K [25] resulting from a rotational transform of $t \approx 1 / 4$ and a finite parallel wave number of turbulent structures [26]. Since the turbulent density fluctuations are associated with potential fluctuations, the ion-saturation current 
spectra $S_{I}(m)_{\mathrm{TPA}}$ and $S_{I}(m)_{\mathrm{OPA}}$ (Fig. 1e and $\mathrm{f}$ ) likewise exhibit maxima in the same spectral range around $m=4$. In contrast to the ion-saturation current spectra, however, most of the power of the potential spectra is found at $m=0$. This poloidally homogeneous potential structure is obviously present at both toroidal positions which indicates toroidal homogeneity as it is characteristic for ZFs.

If the $m=0$ potential fluctuations were related to fluctuations in the equilibrium plasma state, this should also show up in $m=0$ density fluctuations, which are absent. The frequency spectra of the potential signals (not shown here) peak at $3 \mathrm{kHz}$ which is a factor of two lower than expected for a geodesic acoustic mode (GAM) [1], and in discharges with varying ion mass $m_{i}$ and electron temperature $T_{e}$ it was found that the peak frequency does not scale with the ion sound velocity $c_{s}=\sqrt{T_{e} / m_{i}}$. Together with a lack of a prominent $m=1$ mode in the ion-saturation current spectra, we therefore can exclude that the $m=0$ potential perturbation is a GAM. It is pointed out, that due to the number of probes on the flux surface, this is the clearest signature of a ZF obtained in toroidal plasmas so far.
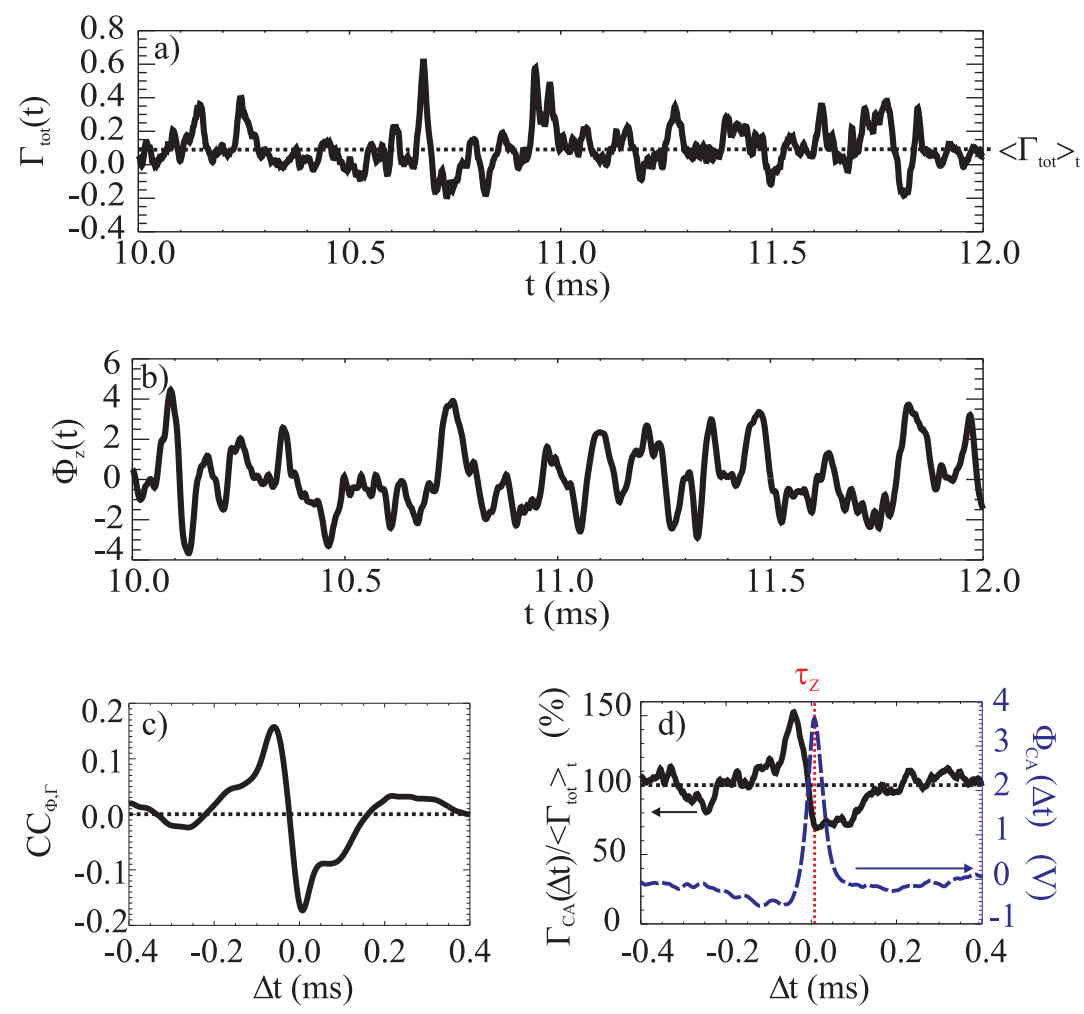

Figure 2. Time series of total turbulent transport (a) and zonal potential (b). Crosscorrelation (c) and conditional averaged transport (solid line) and ZF (blue dashed line) indicate a predator-prey dynamics (d).

In order to study the dynamics of the $m=0$ potential structure and its relation to turbulent transport, the time evolution is examined. Therefor, the total turbulent particle transport $\Gamma_{\text {tot }}(t)=\sum_{j} \Gamma_{\text {loc }}^{j}(t)$ is estimated as the spatial sum over all local transport contributions $\Gamma_{\text {loc }}^{j}(t)$ determined according to Eq. 2 from all probes at both 
arrays. Similarly, the time dependent zonal potential $\Phi_{\mathrm{z}}(t)$ is calculated as the spatial average of all potential signals $\tilde{\phi}_{\mathrm{fl}}(t)$ which is equivalent to the $m=0$ component but a signed quantity. The resulting time traces of the total turbulent transport $\Gamma_{\text {tot }}(t)$ and the zonal potential $\Phi_{\mathrm{z}}(t)$ are shown in Fig. 2a and b, respectively. The turbulent particle transport $\Gamma_{\text {tot }}(t)$ fluctuates on the time scale of turbulence $(\sim 50 \mu \mathrm{s})$ with strong bursts exceeding by fivefold the temporal mean $\left\langle\Gamma_{t o t}\right\rangle_{t}$ (dashed line). Moreover, negative values of the total turbulent transport corresponding to inward transport is repeatedly observed for some microseconds. As it was shown in former works, plasmas in TJ-K are fully dominated by electrostatic drift-wave turbulence [27, 28, 29]. Turbulent diffusivities in TJ-K estimated from a random walk model using measured radial correlation lengths and the lifetime of turbulent structures turn out to be about two orders of magnitude larger than neoclassical ones [30]. Hence, the measured total turbulent particle transport may be identified with the overall global particle transport. Negative values of $\Gamma_{\text {tot }}(t)$ could be interpreted as a transient particle pinch if the contributions of flux surface regions between the probe array positions to transport are assumed to obey the same dynamics as $\Gamma_{\text {tot }}(t)$.

The zonal potential time trace $\Phi_{\mathrm{z}}(t)$ (Fig. 2b) features a bursty behavior similar to the transport, and achieves transitory values of up to $5 \mathrm{~V}$. The bursts have a finite lifetime of some micro seconds pointing to a dynamic which is related to that of turbulence driven ZFs rather than to a pure statistical effect. This is supported by the mixing length estimate of the ZF growth rate $\gamma_{Z F}=\left\langle\tilde{v}_{r}^{2}\right\rangle \tau_{c o r} k_{r}^{2}$ [31] which turns out to be about a factor of two larger than the collisional damping rate for the present situation.

As already found in investigations of the energy transfer between ZF and driftwave turbulence $[7,8,32]$, the predator-prey cycle is also observed here between total turbulent transport $\Gamma_{\text {tot }}(t)$ and ZFs $\Phi_{z}(t)$. This is illustrated in Fig. 2c, where the cross-correlation function

$$
C C_{x y}(\Delta t)=\frac{\langle x(t) y(t+\Delta t)\rangle_{t}}{\sigma_{x} \sigma_{y}}
$$

with $x(t)=\Phi_{\mathrm{z}}(t), y(t)=\Gamma_{\text {tot }}(t)$ and corresponding standard deviations $\sigma\left(\langle\cdot\rangle_{t}\right.$ is a temporal average) shows the typical positive correlation between turbulence and ZF at time lags $\Delta t<0$, and negative correlation at $\Delta t=0$. The latter could be regarded as a consequence of the reduction in turbulence amplitudes by ZFs, which is expected to be reflected in transport, too.

In order to quantify the transport reduction by the ZF, a conditional averaging technique [33] is applied to the time series of total transport and potential. To this end, short time windows with a length of $512 \mu$ s are stored whenever the chosen threshold value of $2 \sigma$ is reached in the $\Phi_{\mathrm{z}}(t)$ time series. Averaging over 781 realizations detected in the potential signal results in $\Phi_{C A}(\Delta t)$, a statistically characteristic picture of the ZF dynamics as depicted in Fig. 2d (blue dashed line). The coherent ZF potential shows up as a burst with a lifetime of about $50 \mu \mathrm{s}$. For the same time windows, the conditionally averaged total transport $\Gamma_{C A}(\Delta t)$ is determined and normalized to the temporal mean 
of total transport $\left\langle\Gamma_{t o t}\right\rangle_{t}$ (Fig. 2d, solid line). The predator-prey behavior as observed in the cross-correlation shows up in the conditional average, too. It can be summarized in a limit-cycle in phase-space (Fig. 3i) as it is theoretically described for the drift-wave and zonal-flow enstrophy [1] and obviously still present between ZF and total turbulent transport. The amplitude of turbulent transport behaves similarly as the drift-wave enstrophy, and its rise prior to the occurrence of the ZF reflects the energy transfer into the ZF. Lowest transport levels are observed, when the ZF reaches maximum values $\left(\Delta t=\tau_{Z}\right)$. Interestingly, the phase of reduced transport lasts for about $100 \mu \mathrm{s}$ and, hence, much longer than the life time of the ZF. In this phase, the total transport is reduced down to $70 \%$ of the temporal mean value, which demonstrates the ability of ZFs to transiently improve global confinement.

But how does the ZF regulate turbulent transport in detail? The Fourier representation of turbulent transport depending on the poloidal wavenumber $k_{\theta}$ reads

$$
\Gamma\left(k_{\theta}\right)=\frac{1}{B}\left|\hat{n}\left(k_{\theta}\right)\right|\left|\hat{E}_{\theta}\left(k_{\theta}\right)\right| \cos \left(\alpha_{n E}\left(k_{\theta}\right)\right)
$$

with the complex (denoted by the hat) density and poloidal electric field amplitudes, $\left|\hat{n}\left(k_{\theta}\right)\right|$ and $\left|\hat{E}_{\theta}\left(k_{\theta}\right)\right|$, and the cosine of the cross phase $\alpha_{n E}\left(k_{\theta}\right)$ between density and electric field. Disregarding the cross phase in Eq. 3, the predator-prey signatures in transport can be attributed to the fluctuation amplitudes, only. The role of the cross phase, however, needs to be clarified. For this purpose, the conditional averaging technique is applied to $k_{\theta}$ spectra of the ion-saturation current and the floating-potential fluctuations which are available at every time point. This way, the characteristic temporal evolution of transport Fourier components can be related to the coherent zonal potential structure from Fig. 2d. The spectra of ion-saturation current $S_{I}\left(k_{\theta}, \Delta t\right)$, floating-potential $S_{\phi}\left(k_{\theta}, \Delta t\right)$, and turbulent transport $\Gamma\left(k_{\theta}, \Delta t\right)$ are shown in Fig. 3a, c, e, respectively. All quantities feature the highest contributions in the range of $k_{\theta}=20$ to $60 \mathrm{~m}^{-1}$ corresponding to turbulent scales around $k_{\theta} \rho_{s}=0.56$ with the drift scale $\rho_{s}=\frac{\sqrt{m_{i} T_{e}}}{e B}$ and $m_{i}$ the ion mass. Between $\Delta t=0$ and $0.15 \mathrm{~ms}$, the contributions of these spectral scales are clearly reduced.

Three selected time points $\tau_{T}$ (high turbulence shortly before the ZF rise), $\tau_{Z}$ (ZF maximum) and $\tau_{R}$ (turbulence reduced after the $\mathrm{ZF}$ ) describing different phases of the ZF (see Fig. 3g) are compared in the following. Prior to the rise of the ZF at $\Delta t=\tau_{T}$, the fluctuation powers and turbulent transport are maximum around $k_{\theta}=40 \mathrm{~m}^{-1}$, and exceed the time averaged spectra (black solid line) shown in Fig. 3b, d, f. This excess of turbulent power gives rise to the growth of the ZF.

In the phase of highest ZF amplitude at $\Delta t=\tau_{Z}$, the ion-saturation current and potential spectra (Fig. 3b, d) are nearly identical to the time averaged spectrum except for the potential $m=0$ peak which is the signature of the ZF. The turbulent transport (Fig. 3f), on the other hand, reaches minimum values at $\Delta t=\tau_{Z}$. The fluctuation amplitudes $\left|\hat{n}\left(k_{\theta}\right)\right|$ and $\left|\hat{E}_{\theta}\left(k_{\theta}\right)\right|$ are therefore ruled out to cause the transport reduction at $\Delta t=\tau_{Z}$. This points to the exceptional role of the cross phase $\alpha_{n E}$ which is shown in Fig. 3j. Indeed, the cross phase deviates significantly from its mean value and has 

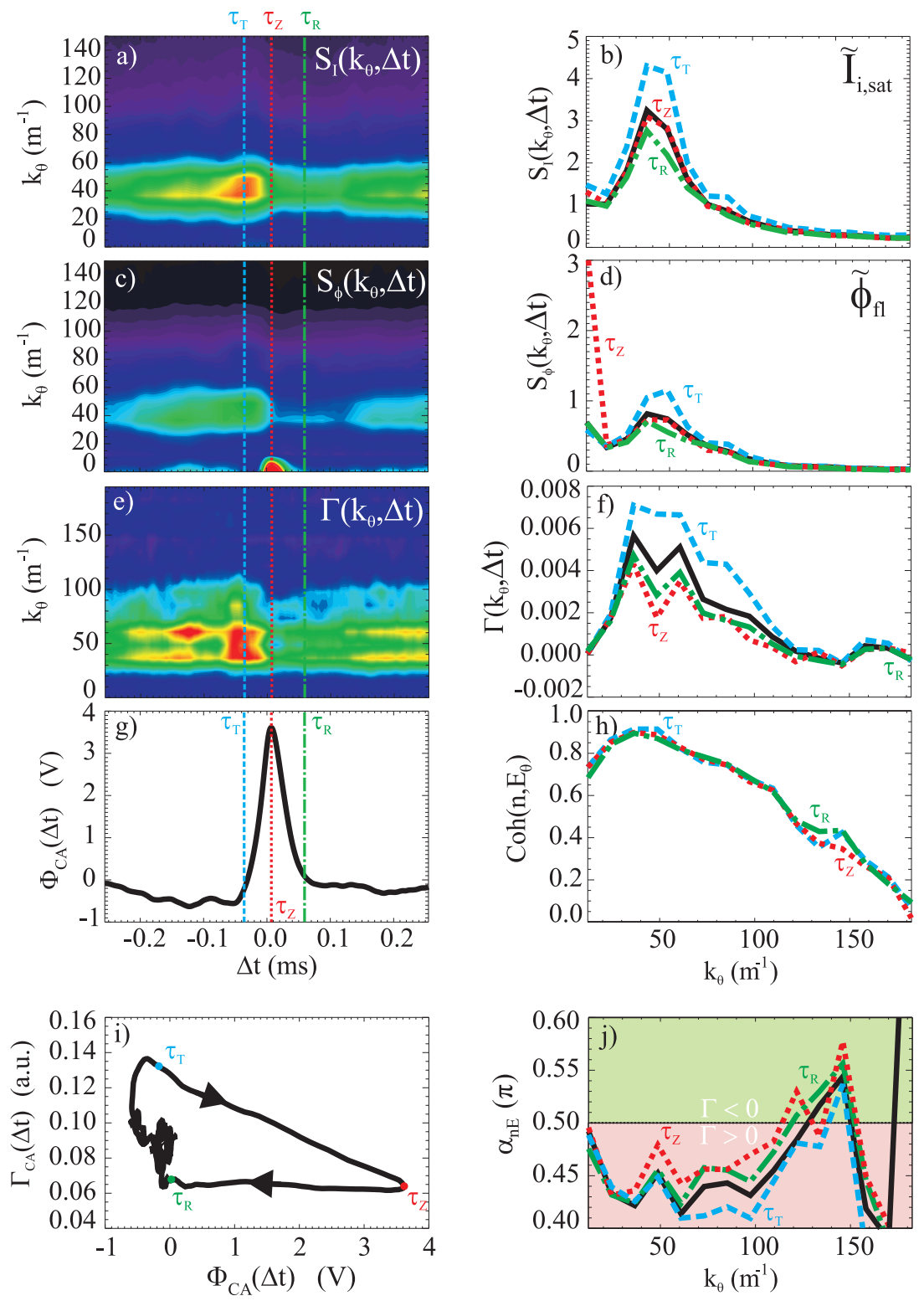

Figure 3. Characteristic evolution of scale resolved ion-saturation current (a, b), floating potential fluctuations (c, d) and turbulent transport (e, f). During different phases of the ZF (g), the coherence $(\mathrm{h})$ and cross phase $(\mathrm{j})$ is shown. In phase-space, $\mathrm{ZF}$ and total turbulent transport establish a limit-cycle (i).

increased values approaching $\alpha_{n E}=0.5$ where the cosine in Eq. 3 reaches zero. Thus, in the early phase of the ZF the cross phase $\alpha_{n E}$ rather than the turbulent amplitudes is relevant for transport reduction as it was already emphasized in theoretical $[34,35]$ works. Also biasing experiments indicated that shear flows mainly act on the cross phase $[36,37,25]$.

Some microseconds later at $\Delta t=\tau_{R}$, the amplitudes are also reduced, and stay on reduced levels until $\Delta t \approx 100 \mu$ s which is twice the ZF life time. Apparently, it takes some time until the turbulence has recovered again after loosing its energy to the 
ZF. The transport, however, starts rising again already at $\Delta t=\tau_{R}$ which is due to the cross phase that has reached almost its mean value. During all considered phases, the cross-coherence (Fig. 3h) as a measure of the reliability of the phase information stood unchanged at reasonably high values in the relevant $k_{\theta}$ range.

In summary, the interaction between ZFs and turbulent transport was measured in great detail. For the first time, the transport reduction by zonal flows was determined quantitatively. A reduction of $30 \%$ was observed in the phase of maximum ZF amplitude which was sustained beyond the lifetime of the ZF. In agreement with theory [34, 35], it was shown that the turbulent transport reduction is initially induced by a modification of the cross phase between density and potential fluctuations and later maintained by reduced fluctuation amplitudes. The findings underline the importance of ZFs as a trigger for global confinement phenomena like the transition from L- to H-mode.

We thank K. Hallatschek and P. Manz for fruitful discussions.

[1] P. H. Diamond, S.-I. Itoh, K. Itoh, and T. S. Hahm, Plasma Phys. Controll. Fusion 47, R35 (2005).

[2] R. H. Kraichnan, Phys. Fluids 10, 1417 (1967).

[3] P. H. Diamond and Y. B. Kim, Phys. Fluids, B 3, 1626 (1991).

[4] B. D. Scott, New Journal of Physics 7, 92 (2005).

[5] P. Xanthopoulos et al., Phys. Rev. Lett. 107, 245002 (2011).

[6] Y. Xiao et al., Physics of Plasmas 17, 022302 (2010).

[7] U. Stroth, Plasmaphysik (Vieweg+Teubner Verlag, Wiesbaden, 2011).

[8] M. Ramisch et al., Plasma Physics and Controlled Fusion 52, 124015 (2010).

[9] P. Manz, M. Ramisch, and U. Stroth, Phys. Rev. Lett. 103, 165004 (2009).

[10] P. Manz, M. Ramisch, and U. Stroth, Plasma Phys. Controll. Fusion 51, 035008 (2009).

[11] A. Fujisawa, Nuclear Fusion 49, 013001 (2009).

[12] P. Manz, G. Birkenmeier, M. Ramisch, and U. Stroth, Physics of Plasmas 19, 082318 (2012).

[13] P. H. Diamond, Y. M. Liang, B. A. Carreras, and P. W. Terry, Phys. Rev. Lett. 72, 2565 (1994).

[14] M. A. Malkov, P. H. Diamond, and M. N. Rosenbluth, Physics of Plasmas 8, 5073 (2001).

[15] M. Sasaki, K. Itoh, S.-I. Itoh, and N. Kasuya, Nuclear Fusion 52, 023009 (2012).

[16] T. Estrada, C. Hidalgo, T. Happel, and P. H. Diamond, Phys. Rev. Lett. 107, 245004 (2011).

[17] L. Schmitz et al., Phys. Rev. Lett. 108, 155002 (2012).

[18] G. D. Conway et al., Phys. Rev. Lett. 106, 065001 (2011).

[19] E.-j. Kim and P. H. Diamond, Phys. Rev. Lett. 90, 185006 (2003).

[20] N. Krause et al., Rev. Sci. Instrum. 73, 3474 (2002).

[21] G. Birkenmeier et al., Phys. Rev. Lett. 107, 025001 (2011).

[22] A. Köhn et al., Plasma Phys. Controll. Fusion 52, 035003 (2010).

[23] S. Enge et al., Phys. Rev. Lett. 105, 175004 (2010).

[24] N. Mahdizadeh et al., Plasma Phys. Controll. Fusion 47, 569 (2005).

[25] M. Ramisch et al., Plasma Phys. Controll. Fusion 49, 777 (2007).

[26] N. Mahdizadeh et al., Plasma Phys. Controll. Fusion 49, 1005 (2007).

[27] U. Stroth et al., Proc. 14th Int. Stellarator Workshop, Greifswald (Germany), September 2003 (IPP, Greifswald, 2003), No. 19, pp. 1-7.

[28] C. Lechte, S. Niedner, and U. Stroth, New J. Phys. 4, 34.1 (2002).

[29] K. Rahbarnia et al., Plasma Phys. Controll. Fusion 50, 085008 (2008).

[30] M. Ramisch et al., Phys. Plasmas 12, 032504 (2005).

[31] P. Manz et al., Physics of Plasmas 19, 012309 (2012).

[32] P. Manz, M. Ramisch, and U. Stroth, Phys. Rev. E 82, 056403 (2010).

[33] H. Johnsen, H. L. Pécseli, and J. Trulsen, Phys. Fluids 30, 2239 (1987).

[34] P. W. Terry, D. E. Newman, and A. S. Ware, Phys. Rev. Lett. 87, 185001 (2001).

[35] A. S. Ware, P. W. Terry, P. H. Diamond, and B. A. Carreras, Plasma Phys. Controll. Fusion 38, 
1343 (1996).

[36] V. Antoni et al., Plasma Phys. Controll. Fusion 42, 83 (2000).

[37] J. A. Boedo et al., Nucl. Fusion 40, 1397 (2000). 